\title{
Electric field gradients of transition metal complexes from density functional theory: assessment of functionals, geometries and basis sets. ${ }^{\dagger, \hbar}$
}

\author{
Ragnar Bjornsson $^{a}$ and Michael Bühl ${ }^{* a}$ \\ Received (in $X X X, X X X)$ Xth $X X X X X X X X X 200 X$, Accepted Xth $X X X X X X X X X 200 X$ \\ ${ }_{5}$ First published on the web $X$ th $X X X X X X X X X 200 X$ \\ DOI: 10.1039/b000000x
}

Revised Version 30 March 2010

\begin{abstract}
Electric field gradients (EFGs) were computed for the first-row transition metal nuclei in $\mathrm{Cr}\left(\mathrm{C}_{6} \mathrm{H}_{6}\right)(\mathrm{CO})_{3}, \mathrm{MnO}_{3} \mathrm{~F}, \mathrm{Mn}(\mathrm{CO})_{5} \mathrm{H}, \mathrm{MnCp}(\mathrm{CO})_{3}, \mathrm{Co}(\mathrm{CO})_{4} \mathrm{H}, \mathrm{Co}(\mathrm{CO})_{3}(\mathrm{NO})$ and $\mathrm{VCp}(\mathrm{CO})_{4}$, for which experimental gas-phase data (in form of nuclear quadrupole coupling constants) are avail10 able from microwave spectroscopy. A variety of exchange-correlation functionals were assessed, among which range-separated hybrids (such as CAM-B3LYP or LC- $\omega$ PBE) perform best, followed by global hybrids (such as B3LYP) and gradient-corrected functionals (such as BP86). While large basis sets are required on the metal atom for converged EFGs, smaller basis sets can be employed on the ligands. In most cases, EFGs show little sensitivity toward the geometrical parameters.
\end{abstract}

\section{Introduction}

In chemistry, gas-phase experiments are indispensible for a number of reasons: Firstly, they furnish fundamental insights into the intrinsic chemistry of molecules unperturbed by a 20 surrounding medium or solvent. ${ }^{1}$ Secondly, they provide key reference data for assessment of computational methods aspiring to understand and predict this chemistry. Such validation is particularly dearly needed in the blooming field of density functional theory (DFT), where the success or 25 failure of a particular exchange-correlation functional is hard to predict beforehand. Systematic DFT performance studies have fertilised computational transition-metal chemistry tremendously, ${ }^{2}$ and will continue to do so as new functionals are constantly being developed.

30

Apart from geometries and energies, spectroscopic properties of transition-metal complexes are important targets for DFT. ${ }^{3}$ A key quantity that can affect such spectroscopic observation (or, in fact, enable it in the first place) is the electric field 35 gradient (EFG) at a quadrupolar nucleus. Any nucleus having spin $I>1 / 2$ has a non-spherical nuclear charge distribution and thus possesses an electric quadrupole moment. The latter can interact with the charge distribution generated by the surrounding nuclei and electrons, an interaction that is 40 governed by the EFG at the nucleus in question. Over $40 \%$ of the stable nuclides of the periodic table are quadrupoles.

The quadrupole interaction is observable through a variety of techniques. The most direct way is through nuclear quadru${ }_{45}$ pole resonance spectroscopy (NQR), but it can also surface in microwave (MW), Mössbauer, electron-nuclear double resonance (ENDOR) and, probably most importantly, NMR spectroscopy. The quadrupole interaction affects the lineshape of NMR signals by providing a relaxation mechanism for the 50 nuclear spin states, resulting in quadrupolar line broadening.
This effect is described by two independent parameters, the nuclear quadrupole coupling constant (NQCC) and the asymmetry parameter, which can both be refined from highresolution anisotropic NMR spectroscopy, usually in the solid 55 state. The NQCC or $\mathrm{C}_{Q}$ is proportional to the product of the nuclear quadrupole moment, $Q$, and the largest component of the EFG, $\mathrm{V}_{\mathrm{zz}}$, as shown in equation 1 .

$$
C_{Q}=\frac{e^{2} Q V_{z z}}{h}
$$

Defined as the second derivative of the electric potential, the ${ }_{60} \mathrm{EFG}$ is a symmetric nine-component traceless tensor, evaluated at the position of each nucleus. Diagonalising the tensor by reorienting in the principal axis system affords the three principal components $\mathrm{V}_{\mathrm{xx}}, \mathrm{V}_{\mathrm{yy}}$ and $\mathrm{V}_{\mathrm{zz}}$ (the diagonal) where $\left|V_{z z}\right| \geq\left|V_{y y}\right| \geq\left|V_{x x}\right|$. The EFG is zero when the charge ${ }_{65}$ distribution around the nucleus has cubic symmetry. In all other cases, the EFG is a sensitive probe into the electronic structure as it depends directly on the electronic and nuclear distribution around the quadrupole. The EFG can thus be utilized to probe structural and chemical features of a 70 molecule or solid whenever the quadrupole interaction parameters can be obtained. Calculation of NQCCs have for example been successfully used to elucidate the structure and enzyme mechanism of transition metal-containing enzyme cofactors where X-ray structures gave an incomplete picture. ${ }^{4}$

Even though the EFG is a first-order property and can be calculated directly as an expectation value, accurate quantumchemical calculations can be quite involved because large basis sets and highly correlated wavefunctions may be 80 needed. ${ }^{5}$ For larger transition metal complexes, DFT is often the only practicable quantum chemical method, MP2 generally performing poorly and higher correlated methods, like coupled cluster, often being out of reach. Quantum chemical calculations of EFG tensors of transition metal ${ }_{85}$ complexes and solids are starting to become common, ${ }^{6}$ but 
few studies have systematically explored the accuracy of the methods that are in use, and these studies have mostly focused on small diatomics. Schwerdtfeger et al. showed that for simple diatomic first-row transition metal (especially copper) 5 compounds, EFG tensors calculated using several common DFT functionals are greatly in error (even yielding the wrong sign of $\mathrm{V}_{\mathrm{zz}}$ ), when compared to both experimental and $\operatorname{CCSD}(\mathrm{T})$ data. $^{7}$ In contrast, calculated EFG tensors of maingroup compounds compare generally well with experiment.

10

As previous studies had mostly focused on diatomic metal compounds (and on the troublesome group 11 of the periodic table, where relativistic effects are particularly pronounced), we felt a study on the performance of DFT methods for 15 calculating EFG tensors of larger metal complexes with saturated ligand shell and less exotic electronic structures was needed. Such a study would allow one to make a better estimate of errors to anticipate from EFG calculations of transition metal complexes with DFT, help in deciding what 20 functional to choose and hopefully shed some light on why transition metal EFG tensors seem to be harder to calculate accurately than main group EFG tensors. In contrast to EFG tensors of group 11 compounds, relativistic effects have been shown to be negligible for ${ }^{57} \mathrm{Fe}$ EFG tensors ${ }^{8}$ and were not 25 considered in our study.

Many experimental studies of the quadrupole interaction in transition metal complexes involve solid-state NMR spectroscopy. While this technique can often accurately determine 30 the EFG parameters, the latter also encode long-range intermolecular effects. As we want to assess the intrinsic accuracy of DFT methods for single molecules, gas-phase experimental data is therefore preferable as reference. For this purpose we have now tested the performance of DFT methods 35 for calculating EFG tensors of first-row transition-metal complexes, for which accurate NQCCs are available from gasphase MW spectroscopy. Special attention is called to the basis-set dependence of calculated EFGs, the importance of the molecular geometry and the accuracy of $a b$ initio methods 40 in comparison with the DFT and experimental results.

\section{Computational details}

Geometries of the metal complexes were optimized at the BP86/AE1 level, i.e. employing the exchange and correlation ${ }_{45}$ functionals of Becke and Perdew, ${ }^{9}$ together with a fine integration grid (75 radial shells with 302 angular points per shell), and an all-electron basis, AE1, consisting of the augmented $8 \mathrm{~s} 7 \mathrm{p} 4 \mathrm{~d}$ Wachters' basis on the metals ${ }^{10}$ and 6$31 \mathrm{G}^{*}$ basis ${ }^{11}$ on all other elements $\left(6-31 \mathrm{G}^{* *}\right.$ was used for the 50 hydride complexes 6 and 7). This level has been shown to reproduce gas-phase electron-diffraction (GED) and MW geometries resonably well. ${ }^{12}$ Geometries optimised with the TPSS functional ${ }^{13}$ (the best performer in that study) and several different basis sets, as well as experimental gas-phase 55 structures were also tested in order to assess the sensitivity of the EFG tensor toward geometrical parameters.
Electric field gradients were calculated using a range of available density functionals available in the Gaussian $03,{ }^{14}$ ${ }_{60}$ Gaussian $09,{ }^{15} \mathrm{NWChem}^{16}$ and ORCA ${ }^{17}$ packages. Functionals comprise LDA, ${ }^{18}$ BP86, ${ }^{9}$ PBE,${ }^{19}$ TPSS, ${ }^{13}$ B3LYP, ${ }^{20}$ PBE0 (hybrid), ${ }^{21}$ TPSSh,${ }^{13}$ M06, ${ }^{22}$ LC- $\omega P B E,{ }^{23}$ CAM-B3LYP,${ }^{24}$ and B2-PLYP. ${ }^{25}$ The basis-set dependence of EFG tensors was also assessed using several basis sets (and mixed combinat${ }_{65}$ ions of them), namely cc-pVTZ ${ }^{26}, 6-311+\mathrm{G}(2 \mathrm{~d}, \mathrm{p})^{27}, 6-31 \mathrm{G}^{* 11}$, $\mathrm{AE} 1$, def2-SVP ${ }^{28}$, def2-TZVPP ${ }^{28}$ and def2-QZVPP ${ }^{28}$ with and without additional diffuse functions from the aug-cc-pVQZ basis set $^{29}$. EFG calculations were also performed with $a b$ initio methods, using the HF, MP2 and CCSD approximations. 70 B2-PLYP and M06 computations were done using ORCA and NWChem, respectively, all others employed the Gaussian suite of programs.

The experimental NQCC data was converted into EFG $V_{z z}$ 75 values using equation 1 and the latest quadrupole moments from Pyykkö. ${ }^{30}$ The $\mathrm{V}_{\mathrm{zz}}$ values in atomic units (au), while not very intuitive, make the experimental data directly comparable towards each other (NQCC data depends on the magnitude of the quadrupole moment of each nuclide as well). ${ }_{80}$ Calculated $\mathrm{V}_{\mathrm{zz}}$ values were compared to experimental $\mathrm{V}_{\mathrm{zz}}$ values and mean absolute (MAE), mean (ME) and maximum errors (MaxE) and slopes of linear regression lines were determined.

${ }_{85}$ It is important to recall ${ }^{31}$ that an opposite sign convention is used in the Gaussian programs for the principal component values of the EFG. We report Gaussian-style $V_{z z}$ values, noting that the sign must be reversed when using equation 1 to compare calculated NQCCs with MW data. 90

\section{Results and discussion}

The set of first-row transition metal complexes, for which experimental gas-phase NQCC data are available, is displayed in Scheme 1.

95
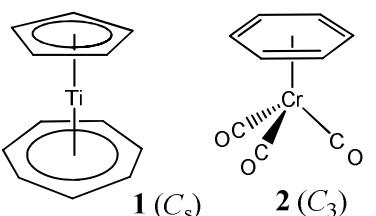

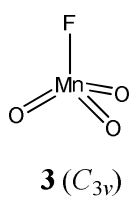<smiles>O=[W][14C](=O)[14C](=O)[O-]</smiles>
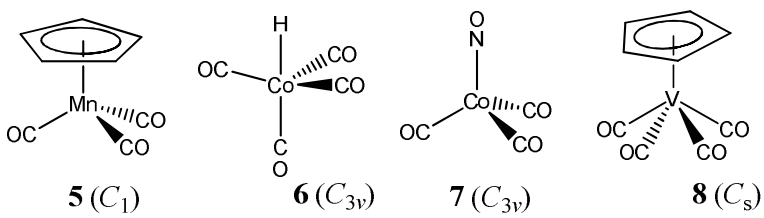

Scheme 1: Test set for EFGs of first-row transition-metal complexes

100 EFGs calculated with different functionals (using BP86/AE1 geometries with the large def2-QZVPP basis set) are collected in Table 1. When comparing these data (and all subsequently calculated ones) to experiment, an unusually large dis 
Table 1. EFG calculations with different functionals: $V_{z z}$ values in au, ${ }^{a}$ compared to gas-phase MW data.

\begin{tabular}{|c|c|c|c|c|c|c|c|c|c|c|c|c|}
\hline Comp. & LDA & BP86 & PBE & TPSS & B3LYP & PBE0 & TPSSh & M06 & $\begin{array}{l}\text { LC- } \\
\omega \mathrm{PBE}\end{array}$ & $\begin{array}{l}\text { CAM- } \\
\text { B3LYP }\end{array}$ & $\begin{array}{l}\text { RI-B2- } \\
\text { PLYP }\end{array}$ & Exp. \\
\hline 1 & -0.790 & -0.726 & -0.714 & -0.719 & -0.775 & -0.765 & -0.737 & -0.558 & -0.866 & -0.842 & -0.781 & $-0.115^{32}$ \\
\hline 2 & -0.348 & -0.326 & -0.316 & -0.322 & -0.339 & -0.329 & -0.328 & -0.388 & -0.433 & -0.380 & -0.600 & $-0.344^{33}$ \\
\hline 3 & -0.569 & -0.567 & -0.554 & -0.514 & -0.451 & -0.395 & -0.465 & -0.511 & -0.280 & -0.359 & -0.115 & $-0.217^{34}$ \\
\hline 4 & 0.500 & 0.502 & 0.495 & 0.499 & 0.533 & 0.544 & 0.514 & 0.565 & 0.566 & 0.553 & 0.555 & $0.570^{35}$ \\
\hline 5 & -0.782 & -0.758 & -0.730 & -0.742 & -0.812 & -0.817 & -0.773 & -0.874 & -0.910 & -0.869 & -0.839 & $-0.877^{36}$ \\
\hline 6 & -0.811 & -0.880 & -0.869 & -0.926 & -1.123 & -1.165 & -1.082 & -1.258 & -1.164 & -1.212 & -0.407 & $-1.182^{37}$ \\
\hline 7 & -0.423 & -0.401 & -0.400 & -0.398 & -0.478 & -0.520 & -0.442 & -0.538 & -0.581 & -0.541 & -0.466 & $-0.356^{38}$ \\
\hline 8 & -0.378 & -0.340 & -0.337 & -0.331 & -0.294 & -0.281 & -0.326 & -0.331 & -0.289 & -0.285 & -0.211 & $-0.390^{39}$ \\
\hline MAE $^{b}$ & 0.139 & 0.136 & 0.142 & 0.126 & 0.088 & 0.081 & 0.096 & 0.095 & 0.076 & 0.075 & 0.211 & \\
\hline $\mathrm{ME}^{\mathrm{b}}$ & -0.002 & 0.004 & 0.012 & 0.009 & -0.024 & -0.024 & -0.015 & -0.077 & -0.042 & -0.043 & 0.102 & \\
\hline $\operatorname{MaxE}^{\mathrm{b}}$ & -0.371 & 0.351 & 0.337 & 0.297 & 0.234 & 0.178 & 0.248 & 0.295 & 0.225 & 0.185 & -0.775 & \\
\hline Slope ${ }^{b}$ & 0.741 & 0.767 & 0.753 & 0.787 & 0.912 & 0.942 & 0.874 & 0.994 & 0.985 & 0.982 & 0.649 & \\
\hline
\end{tabular}

${ }^{\mathrm{a}}$ Gaussian-style, QZVPP basis and BP86/AE1 geometries.

${ }^{b}$ Mean absolute error (MAE), mean error (ME), maximum error (MaxE) and slope from a linear regression of DFT vs. s experimental data (excluding complex 1, see text).

crepancy became apparent for the Ti complex 1. Irrespective of the level of theory, the computed $\mathrm{V}_{\mathrm{zz}}$ values are larger than the experimental ones by roughly a whole order of 10 magnitude.A reinvestigation of the original measurements is underway. ${ }^{40}$ Trusting that this discord will be sorted out eventually, we have omitted the data for $\mathbf{1}$ from the statistical analysis. They are, nevertheless, included at selected levels in the tables for future reference.

The EFG results with the local density approximation (LDA) are interestingly in reasonable agreement with experiment, much better than the Hartree-Fock data (see later). Curiously, the generalized-gradient approximation (GGA) does not

20 improve upon LDA. The BP86 functional is marginally better while the PBE results are even slightly worse (compare, for instance, the mean absolute errors, MAE, in Table 1). The meta-GGA approximation exemplified by the TPSS functional performs slightly better than LDA.

25

The (global) hybrid functionals B3LYP, PBE0 and TPSSh, on the other hand, give considerably better results than their parent GGA variants. We also considered the newer rangeseparated hybrid functionals. The long-range corrected LC-

$30 \omega \mathrm{PBE}$, that has the correct asymptotic behaviour of the exchange interaction, and the parameterized range-separated CAM-B3LYP hybrid functional give similar results and are in fact the best performers of all the DFT methods. For LC$\omega P B E$ and CAM-B3LYP, the observed $V_{z z}$ values are 35 reproduced with a MAE of $0.075-0.076 \mathrm{au}$, or ca. $4 \%$ of the total range covered (1.75 au). Several other DFT functionals were also considered, and the data can be found as electronic supplementary material (ESI). The results for the functionals in Table 1 can be considered to be representative for their 40 respective families: i.e. LDA, GGA, meta-GGA, global and range-separated hybrids. The relative performance of selected functionals is illustrated in Figure 1.

Errors in EFG tensors for both the $\mathrm{Cu}$ and $\mathrm{Cl}$ atoms in $\mathrm{CuCl}$ 45 were found to correlate with errors in the dipole moment using the tested $a b$ initio and DFT methods. ${ }^{7}$ It was argued that several DFT methods may incorrectly describe the charge distribution leading to the EFG errors and that this may mostly be due to shortcomings of the exchange functional. As 50 the total charge has to be conserved, even the long-range exchange interaction may be of importance for an accurate description of the EFG tensor. This argument may serve as rationalisation for the good performance of the non-local hybrid functionals. On the other hand, the double-hybrid 55 functional B2-PLYP performs rather poorly (last DFT entry in Table 1), despite the inclusion of non-local correlation.

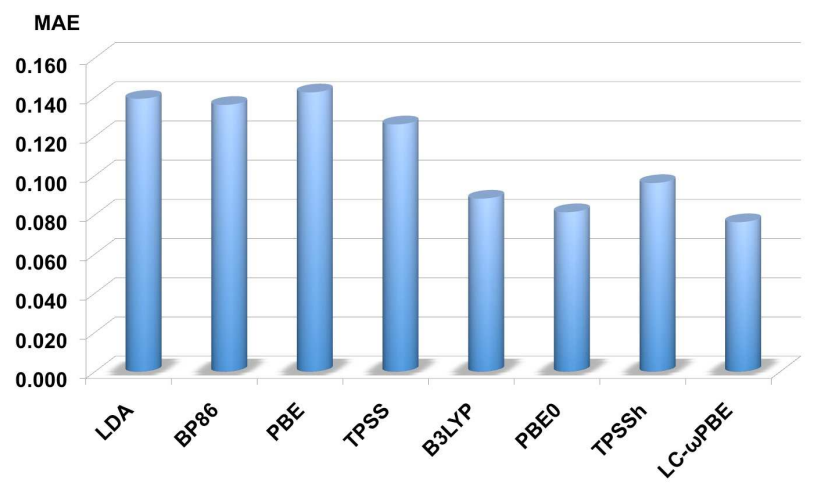

Figure 1: Histogram of the mean absolute errors in DFT${ }_{60}$ computed EFGs $\left(\mathrm{V}_{\mathrm{zz}}\right.$ values in a.u.) for selected functionals from Table 1 .

The QZVPP basis on all atoms is quite large and should be close to the DFT basis set limit Such extended basis sets are, ${ }_{65}$ however, not feasible in practice for larger systems. Searching 
Table 2. B3LYP-computed EFGs ( $\mathrm{V}_{\mathrm{zz}}$ values in au) using different basis sets, ${ }^{\text {a }}$ together with the mean absolute error (MAE) $)^{\mathrm{b}}$ with respect to aug-QZVPP (in parentheses: total number of basis functions for complex $\mathbf{5}$ ).

\begin{tabular}{|c|c|c|c|c|c|c|c|c|c|c|}
\hline Comp. & $\begin{array}{r}\text { aug-QZVPP } \\
(1268) \\
\end{array}$ & $\begin{array}{r}\text { QZVPP } \\
(877) \\
\end{array}$ & $\begin{array}{r}\text { QZVPP/ } \\
\text { TZVPP } \\
(511) \\
\end{array}$ & $\begin{array}{r}-Q Z V P P / \\
\text { SVP } \\
(279) \\
\end{array}$ & $\begin{array}{r}\text { QZVPP/ } \\
6-31 G^{*} \\
(264) \\
\end{array}$ & $\begin{array}{r}\text { Wachters/ } \\
6-31 \mathrm{G}^{*} \\
(213) \\
\end{array}$ & $\begin{array}{r}\text { TZVPP } \\
(475) \\
\end{array}$ & $\begin{array}{r}\text { TZVPP/ } \\
6-31 G^{*} \\
(228) \\
\end{array}$ & $\begin{array}{r}6-311+ \\
G(2 d, p) \\
(392) \\
\end{array}$ & $\begin{array}{r}\text { cc-pVTZ } \\
(468) \\
\end{array}$ \\
\hline 1 & -0.781 & -0.775 & -0.776 & -0.798 & -0.809 & -1.056 & -0.887 & -0.904 & -1.031 & -0.774 \\
\hline 2 & -0.344 & -0.339 & -0.340 & -0.330 & -0.338 & -0.402 & -0.409 & -0.409 & -0.404 & -0.304 \\
\hline 3 & -0.411 & -0.451 & -0.441 & -0.338 & -0.333 & -0.392 & -0.328 & -0.342 & -0.375 & -0.335 \\
\hline 4 & 0.523 & 0.533 & 0.522 & 0.522 & 0.529 & 0.621 & 0.550 & 0.554 & 0.649 & 0.577 \\
\hline 5 & -0.814 & -0.812 & -0.809 & -0.802 & -0.795 & -0.960 & -0.903 & -0.909 & -0.965 & -0.809 \\
\hline 6 & -1.183 & -1.123 & -1.136 & -1.134 & -1.180 & -1.384 & -1.175 & -1.170 & -1.379 & -1.163 \\
\hline 7 & -0.457 & -0.478 & -0.469 & -0.477 & -0.483 & -0.649 & -0.554 & -0.548 & -0.599 & -0.521 \\
\hline 8 & -0.291 & -0.294 & -0.285 & -0.269 & -0.244 & -0.323 & -0.318 & -0.325 & -0.304 & -0.233 \\
\hline MAE & & 0.020 & 0.015 & 0.027 & 0.026 & 0.106 & 0.056 & 0.057 & 0.103 & 0.045 \\
\hline
\end{tabular}

${ }^{a}$ Notation "basis on metal/basis on ligands". ${ }^{b}$ Excluding 1.

5

for more economical basis-set combinations, we carried out additional EFG calculations with the B3LYP functional. The results obtained with a variety of basis sets are summarised in Table 2. With additional diffuse functions on all atoms, ${ }_{10} \mathrm{~B} 3 \mathrm{LYP} / \mathrm{aug}-\mathrm{QZVPP} \quad \mathrm{V}_{\mathrm{zz}}$ values were used as reference. Judging from the MAE values, a quadruple-zeta basis set on the metal atom is important for results close to the B3LYP/aug-QZVPP reference values, but a small double-zeta basis set like 6-31G* is mostly sufficient for the ligand atoms.

15 The mixed QZVPP/6-31G* basis set is not much larger than the AE1 basis (Wachters/6-31G*), containing just an additional 51 basis functions on the metal, but gives results much closer to the reference values. Despite being highly unbalanced, this mixed basis set thus emerges as a cost20 effective compromise for EFG computations for metal centers in transition-metal complexes. This result is reminiscent of NMR chemical shift computations, where "locally dense" basis sets are well established, which consist of extended basis sets on the NMR nuclei of interest, and more compact 25 ones on all other atoms. ${ }^{41}$ A triple-zeta basis set on all atoms, like $6-311+G(2 d, p)$, can have many more basis functions than the mixed QZVPP/6-31G* basis set, but gives unsatisfactory results. As a rough guide for the size of a given basis-set combination, the number of basis functions for a tyical 30 medium-sized complex, $\mathbf{5}$ in this case, is included in Table 2 (see numbers in parentheses in the header). As expected, substituting the inner core electrons of the metal with an effective core potential gives unacceptable results for the EFG tensor for many of the metal complexes (results not shown).

35

The chemical shift tensor is known to be sensitive to the geometry employed in a calculation. In order to probe for the geometry dependence of EFGs, we have evaluated them at the B3LYP/QZVPP level using geometries from different sources 40 (Table 3). Changing the geometry from BP86/AE1 to TPSS/AE1 has negligible effects on the MAE, while slightly improving the slope. Further enlarging the basis set used in the TPSS optimisations to TZVPP and QZVPP on all atoms has also very small effects on the EFG. Using the experiment-
${ }_{45}$ Table 3. B3LYP/QZVPP $V_{z z}$ values (in au), computed for different geometries and compared to gas-phase MW data. ${ }^{a}$

\begin{tabular}{|c|c|c|c|c|c|c|}
\hline \multicolumn{2}{|c|}{ /geom. BP86/ } & \multirow{2}{*}{$\begin{array}{l}\text { TPSS/ } \\
\text { AE1 }\end{array}$} & \multirow{2}{*}{$\begin{array}{l}\text { TPSS/ } \\
\text { TZVPP }\end{array}$} & \multirow{2}{*}{$\begin{array}{l}\text { TPSS/ } \\
\text { QZVPP }\end{array}$} & \multirow[b]{2}{*}{ Expt. $^{b}$} & \multirow[b]{2}{*}{ Ref } \\
\hline Comp. & AE1 & & & & & \\
\hline 2 & -0.339 & -0.324 & -0.320 & -0.322 & -0.288 & 42 \\
\hline 3 & -0.451 & -0.451 & -0.451 & -0.461 & -0.489 & 34 \\
\hline 4 & 0.533 & 0.560 & 0.534 & 0.538 & 0.117 & 35 \\
\hline 5 & -0.812 & -0.802 & -0.804 & -0.805 & -0.793 & 43 \\
\hline 6 & -1.123 & -1.129 & $-1.154^{c}$ & -1.154 & -1.253 & 37 \\
\hline 7 & -0.478 & -0.465 & $-0.466^{\mathrm{d}}$ & -0.452 & -0.399 & 44 \\
\hline 8 & -0.294 & -0.273 & -0.257 & -0.256 & -0.252 & 45 \\
\hline $\mathrm{MAE}^{\mathrm{c}}$ & 0.088 & 0.088 & 0.091 & 0.090 & 0.159 & \\
\hline $\mathrm{ME}^{\mathrm{c}}$ & -0.024 & -0.013 & -0.017 & -0.016 & -0.080 & \\
\hline $\operatorname{MaxE}^{\mathrm{c}}$ & 0.234 & 0.235 & 0.234 & 0.244 & 0.453 & \\
\hline Slope ${ }^{c}$ & 0.912 & 0.927 & 0.925 & 0.926 & 0.741 & \\
\hline
\end{tabular}

${ }^{\mathrm{a}}$ See Table 1. ${ }^{\mathrm{b}}$ Using experimental gas-phase geometries from the given references. ${ }^{\mathrm{c}}$ See footnote $\mathrm{b}$ in Table 1.

50

al geometries from the MW or GED studies, rather than DFToptimised ones, ${ }^{\#}$ affords only minor changes in most cases (compare, e.g. TPSS/QZVPP and Expt entries in Table 3). For the Mn hydride 4, however, the result is significantly worse, ${ }_{55}$ producing an error of 0.45 au (cf. the corresponding MaxE value), which causes the overall statistics to deteriorate notably (see ME and MAE values). In the case of $\mathbf{4}$ the optimised geometries, e.g. $r(\mathrm{Mn}-\mathrm{H}), r\left(\mathrm{Mn}-\mathrm{C}^{\mathrm{ax}}\right)$ and $r\left(\mathrm{Mn}-\mathrm{C}^{\mathrm{eq}}\right)$ of $1.58 \AA, 1.85 \AA$ and $1.85 \AA$, respectively (TPSS/QZVPP 60 level), are probably more accurate than the MW-derived one, which is also associated with larger uncertainties, cf. $r(\mathrm{Mn}-$ $\mathrm{H}), r\left(\mathrm{Mn}-\mathrm{C}^{\mathrm{ax}}\right)$ and $r\left(\mathrm{Mn}-\mathrm{C}^{\mathrm{eq}}\right)$ of 1.65(2) $\AA, 1.82(2) \AA$ and 1.86 $\AA$, respectively. ${ }^{35}$ In general, however, EFG tensors of metal centres seem to be quite insensitive to such geometrical ${ }_{65}$ details, at least for the complexes of this study. Thus it seems unlikely that, for instance, ro-vibrational corrections, which lead from equilibrium to thermally averaged geometries, 
would affect computed EFGs significantly.

In order to assess conventional ab initio methods for our test set, we also evaluated EFG tensors at the Hartree-Fock 5 (HF)/QZVPP, MP2/QZVPP and coupled-cluster singles and doubles (CCSD)/QZVPP/6-31G** levels. These results are included in the ESI. Not unexpectedly, the HF method performs poorly for these first-row transition-metal complexes, with a MAE of 0.32 au, i.e. considerably worse than LDA 10 (0.14 au, Table 1). Even though inclusion of some HF exchange is beneficial for the DFT calculations, exchange alone (HF) clearly clearly cannot give a sufficient description of the EFG. MP2 correlation does not improve the agreement with experiment, in fact the MP2 results can be even worse 15 than HF.

CCSD calculations scale very unfavorably with basis-set size, and the largest basis set that could be used for all molecules was a mixed basis set with QZVPP on the metal and 6-31G** 20 on the ligand atoms. Selected test calculations with larger basis sets on the ligands show that this smaller basis is not properly converged. Moreover, the so-called T1 diagnostic ${ }^{46}$ at the CCSD/QZVPP/6-31G** level affords values in excess of 0.027 throughout, suggesting considerable multireference 25 character for all of the metal complexes. ${ }^{\$}$ This is particularly pronounced for $\mathrm{MnO}_{3} \mathrm{~F}(4, \mathrm{~T} 1=0.10)$, the multireference character of which has been investigated before. ${ }^{47}$ In view of these limitations it is not surprising that the CCSD/QZVPP/6$31 \mathrm{G}^{* *}$ results are in worse agreement with experiment (MAE $300.12 \mathrm{au}$ ) than those from the hybrid DFT variants. Thus, the latter are clearly the methods of choice for the computation of EFGs in transition-metal complexes.

Before the final assessment, a brief discussion of experimental 35 errors is in order. While the NQCCs extracted from highresolution MW spectra are usually very precise, the EFG values derived via eq (1) are associated with larger uncertainties stemming from the nuclear quadrupole moments $Q$. For ${ }^{53} \mathrm{Cr}$ in particular, these uncertainties can amount to ca. $30 \%$ 40 of the actual value of $Q .{ }^{30}$ Thus, perfect agreement between experimentally derived and theoretical $\mathrm{V}_{\mathrm{zz}}$ values is not to be expected.

\section{Conclusions}

${ }_{45} \mathrm{We}$ have validated DFT methods for the first-principles computation of electric field gradients (EFGs) of the metal centres in complexes from the first transition row. For this purpose we selected a compact test set of seven molecules, for which experimental gas-phase data (in form of nuclear 50 quadrupole coupling constants) are available from microwave spectroscopy. A variety of exchange-correlation functionals and basis sets were assessed against these experimental EFGs.

A clear trend was observed for the different classes of 55 functionals, with little distinction between individual members of these classes. Overall, range-separated hybrids (such as CAM-B3LYP or LC- $\omega$ PBE) perform best, followed by global hybrids (such as B3LYP) and gradient-corrected functionals (such as BP86) or meta-GGAs (such as TPSS) and ${ }_{60}$ LDA. While large basis sets are required on the metal centre for converged EFGs, smaller basis sets can be employed on the ligands. Computed EFGs show little sensitivity toward the geometrical parameters. Because of substantial multireference character of the wavefunctions, conventional (single65 reference) $a b$ initio methods (HF, MP2, CCSD) should not be used.

In summary, our recommended protocol for EFG computations of transition metal complexes involves geometry 70 optimisation at an appropriate level (such as BP86 or TPSS/AE1), followed by the property evaluation using LC$\omega$ PBE or CAM-B3LYP (or B3LYP) and a mixed, "locally dense" basis such as QZVPP/6-31G*.

75 Unfortunately, a similar validation for second- or third-row transition metal complexes is hampered by a dearth of experimental NQCC values for such complexes in the gas phase. More, accurate gas-phase data is highly desirable for this class of compounds.

80

Acknowledgement: This work was supported by EaStChem via the EaStChem Research Computing facility and a local Opteron PC cluster maintained by Dr. H. Früchtl.

\section{Notes and references}

${ }_{85}{ }^{a}$ School of Chemistry, North Haugh, University of St. Andrews, St. Andrews, Fife, UK, KY16 9ST. E-mail: buehl@st-andrews.ac.uk

\$ Dedicated to Prof. Dr. D. W. H. Rankin on the occasion of his retirement.

$\dagger$ Electronic Supplementary Information (ESI) available: Additional 90 tabular material and full citations for references 14,15,16. See DOI: $10.1039 / \mathrm{b} 000000 \mathrm{x} /$

\# We used optimised rather than experimental gas-phase geometries for most of our assessments, because in general applications the former, rather than the latter will be more readily accessible.

95 \$ A T1 value of 0.02 is the commonly accepted threshold, above which the wavefunction is indicated to lose its single-reference character, so that regular coupled-cluster theory may not be applicable any more (cf. reference 46).

1 E.g. N. W. Mitzel and D. W. H. Rankin, Dalton Trans. 2003, 3650; D. Hnyk and D. W. H. Rankin, Dalton Trans. 2009, 585.

2 T. Ziegler, Can. J. Chem., 1995, 73, 743.

3 F. Neese, Coord. Chem. Rev., 2008, 253, 526.

4 N. R. Renirie, Z. Hasan, R. Wever, A. J. Vega and T. Polenova, ,J. Am. Chem. Soc. 2006, 128, 5190; M. P. Waller, M. Bühl, K. R. Geethalakshmi, D. Wang and W. Thiel, Chem. Eur. J., 2007, 13, 4723; M. P. Waller, K. R. Geethalakshmi and M. Bühl, PooransinghMargolis, J. Phys. Chem. B, 2008, 112, 5813; K. R. Geethalakshmi, M. P. Waller and W. Thiel, M. Bühl, J. Phys. Chem. B, 2009, 113, 4456; K. V. Narasimhulu, R. Carmieli and D. Goldfarb, J. Am. Chem. Soc., 2007,129, 5391; A. S. Lipton, R. W. Heck and P. D. Ellis, J. Am. Chem. Soc., 2004, 126, 4735; A. S. Lipton, R. W. Heck, W. A. de Jong, A.R. Gao, X. Wu, A. Roehrich, G. S. Harbison and P. D. Ellis, J. Am. Chem. Soc. 2009, 131, 13992.

5 P. Schwerdtfeger, M. Pernpointner and J. K. Laerdahl, J. Chem. Phys., 1999, 111, 3357; P. Schwerdtfeger, R. Bast, M. C. Gerry, C. R. Jacob, M. Jansen, V. Kellö, A. V. Mudring, A. J. Sadlej, T. Saue et al., J. Chem. Phys., 2005, 122, 124317; V. Kellö, A. J. Sadlej, P. Pyykkö, D. Sundholm and M. Tokman, Chem. Phys. Lett., 1999, 304, 414-422. 
6 In context with NMR see e.g. . Bühl, Ann. Rep. NMR Spectrosc. 2008, 64, 77; J. Cuny, S. Messaoudi, V. Alonzo, E. Furet, J. F. Halet, E. Le Fur, S. E. Ashbrook, C. J. Pickard, R. Gautier and L. Le Polles, J. Comput. Chem., 2008, 29, 2279.

7 P. Schwerdtfeger, M. Pernpointner and J. K. Laerdahl, J. Chem. Phys., 1999, 111, 3357; R. Bast and P. Schwerdtfeger, J. Chem. Phys., 2003, 119, 5988; C. Thierfelder, P. Schwerdtfeger and T. Saue, Phys. Rev. A, 2007, 76, 34502.

8 S. Sinnecker, L. D. Slep, E. Bill and Frank Neese, Inorg. Chem., 2005, 44, 2245.

9 A. D. Becke, Phys. Rev. A 1988, 38, 3098; J. P. Perdew, Phys. Rev. B 1986, 33, 8822; J. P. Perdew, Phys. Rev. B 1986, 34, 7406.

10 A. J. H. Wachters, J. Chem. Phys. 1970, 52, 1033; P. J. Hay, J. Chem. Phys. 1977, 66, 4377.

11 W. J. Hehre, R. Ditchfield and J. A. Pople, J. Chem. Phys. 1972, 56, 2257; P. C. Hariharan and J. A. Pople, Theor. Chim. Acta. 1973, 28, 213.

12 M. Bühl and H. Kabrede, J. Chem. Theory Comput. 2006, 2, 1282.

13 J. Tao, J. P. Perdew, V. N. Staroverov and G. E. Scuseria, Phys. Rev. Lett., 2003, 91, 146401.

14 M. J. Frisch, J. A. Pople et al., Gaussian 03, Revision E.01, Gaussian, Inc., Pittsburgh PA, 2003.

15 Frisch, M. J. et al., Gaussian 09, Revision A.02, Gaussian, Inc., Wallingford CT, 2009.

16 E. J. Bylaska, W. A. de Jong et al., NWChem 5.1. Pacific Northwest National Laboratory, Richland, Washington, 2007.

17 F. Neese, ORCA - an Ab initio, Density Functional and Semiempirical Program Package, 2.7-Rev. 0; Universität Bonn: Bonn, Germany, 2009.

18 P. Hohenberg and W. Kohn, Phys. Rev., 1964, 136, B864; W. Kohn and L. J. Sham, Phys. Rev., 1965, 140, A1133; J. C. Slater, The SelfConsistent Field For Molecular and Solids, Quantum Theory of Molecular and Solids, 1974, 4; S. H. Vosko, L. Wilk and M. Nusair, Can. J. Phys., 1980, 58, 1200.

19 J. P. Perdew, K. Burke, and M. Ernzerhof, Phys. Rev. Lett., 1996, 77, 3865; J. P. Perdew, K. Burke, and M. Ernzerhof, Phys. Rev. Lett., 1997, 78, 1396.

20 A. D. Becke J. Chem. Phys. 1993, 98, 5648; C. Lee, W. Yang, R. G. Parr, Phys. Rev. B, 1988, 37, 785.

21 C. Adamo and V. Barone, J. Chem. Phys. 1999, 110, 6158.

22 Y. Zhao and D. G. Truhlar, Theor. Chem. Acct. 2008, 120, 215.

23 O. A. Vydrov, J. Heyd, A. V. Krukau and G. E. Scuseria, J. Chem. Phys., 2006, 125, 074106; O. A. Vydrov and G. E. Scuseria, J. Chem. Phys., 2006, 125, 234109.

24 T. Yanai, D. P. Tew and N. C. Handy, Chem. Phys. Lett., 2004, 393, 51.

25 S. Grimme, J. Chem. Phys., 2006, 124, 034108; T. Schwabe and S. Grimme, Phys. Chem. Chem. Phys., 2006, 8, 4398.

26 T. H. Dunning, J. Chem. Phys., 1989, 90, 1007.

27 R. Krishnan, J. S. Binkley, R. Seeger and J. A. Pople, J. Chem. Phys., 1980, 72, 650; A. J. H. Wachters, J. Chem. Phys., 1970, 52, 1033; P. J. Hay, J. Chem. Phys., 1977, 66, 4377.

28 F. Weigend and R. Ahlrichs, Phys. Chem. Chem. Phys., 2005, 7, 3297.

29 T.H. Dunning, Jr., J. Chem. Phys., 1989, 90, 1007; R.A. Kendall, T.H. Dunning, Jr. and R.J. Harrison, J. Chem. Phys., 1992, 96, 6796; N.B. Balabanov and K.A. Peterson, J. Chem. Phys., 2005, 123, 064107.

30 P. Pyykkö, Mol. Phys. 2008, 106, 1965.

31 S. Adiga, D. Aebi and D. L. Bryce, Can. J. Chem., 2007, 85, 496.

32 K. S. Keck, C. Tanjaroon and S. G. Kukolich, J. Mol. Spectr., 2005, 232, 55.

33 S. G. Kukolich, S. M. Sickafoose, L. D. Flores and S. Beckenridge, J. Chem. Phys., 1994, 100, 6125.

34 A. Javan and A. Engelbrecht, Phys. Rev., 1954, 96, 649.

35 S. G. Kukolich and S. M. Sickafoose, Inorg. Chem., 1994, 33, 1217.

36 M. A. Roehrig, D. W. Wallace and S. G. Kukolich, J. Chem. Phys., 1992, 96, 2449.

37 S. G. Kukolich and S. M. Sickafoose, J. Chem. Phys., 1996, 105, 3466 .
38 S. G. Kukolich, M. A. Roehrig, S. T. Haubrich and J. A. Shea, J. Chem. Phys., 1991, 94, 191.

39 R. T. Kay, J. L. Hubbard and S. G. Kukolich, J. Mol. Spectr., 1995, 172, 378 .

40 S. G. Kukolich, private communication.

41 D. B. Chesnut, B.E. Rusiloski, K.D. Moore and D. A. Egolf, J. Comput. Chem, 1993, 14, 1364.

42 S. M. Sickafoose, S. M. Beckenridge and S. G. Kukolich, Inorg. Chem., 1994, 33, 5176.

43 GED: M. J. Almond, E. M. Page, D. A. Rice, K. Hagen and H. V. Volden, J. Mol. Struct., 1994, 319, 223.

44 GED: L. Hedberg, K. Hedberg, S. K. Satija and B. I. Swanson, Inorg. Chem., 1985, 18, 2766.

45 GED: M. J. Almond, E. M. Page, D. A. Rice and K. Hagen, J. Organomet. Chem., 1996, 511, 303.

46 T. J. Lee and P. R. Taylor, Int. J. Quantum Chem. Symp. 1989, 23, 199.

47 H. Bürger, P. Weinrath, S. Dressler, T. Hansen and W. Thiel, J. Mol. Spectr., 1997, 183, 139. 